\title{
Prôner la concurrence ne résout rien!
}

Toutes les études le confirment: la pénurie de médecins ne frappe pas que l'Allemagne ou la France, mais la Suisse également, et de manière draconienne. Face à ce constat, comment comprendre le sens du projet visant à limiter le nombre des prestataires du domaine de la santé, en premier lieu les médecins? Dans le courant de l'été dernier, le Conseil fédéral a introduit la planification des besoins en matière d'installation de nouveaux cabinets médicaux. Il convient dès lors d'attendre les résultats de cette décision avant de prendre d'autres mesures légales ou administratives irréfléchies.

La formule magique du moment a pour nom «concurrence»: la liberté contractuelle serait en effet à même de produire des économies. Mais une concurrence comment et pour quoi? Certes, le principe de concurrence peut diminuer le coût des prestations mais pas dans le but de diminuer la quantité des prestations vendues, bien plutôt dans celui d'augmenter le chiffre d'affaires et les quantités. Ce sont précisément ces quantités et non les prix qui renchérissent la santé publique.

La concurrence devrait avoir, du moins peuton l'espérer, un effet bénéfique sur la qualité. Mais comment donc mesurer - la satisfaction du patient mise à part - la qualité objective, essentielle, du succès thérapeutique à long terme, et qui devra en définir les critères? Le domaine de la santé publique en Suisse ne dispose pas des données nécessaires en la matière et leur saisie pourrait, au mieux, prendre des années. On ne saurait pallier cette lacune en confiant l'entière responsabilité de cette évaluation aux assureurs, abstraction faite que ces derniers ne seraient guère en mesure de l'assumer. La FMH rejette dès lors l'attribution de cette responsabilité aux seuls assureurs. Une telle appréciation est de prime abord du ressort des organisations médicales compétentes, qui reçoivent pour ce faire un mandat du Conseil fédéral.

Si le projet actuel de la Commission de la sécurité sociale et de la santé publique du Conseil national (CSSS-N) était adopté, près de $30 \%$ des médecins seraient soumis à une procédure en raison du caractère non économique de leurs prestations: une aberration manifeste et hors de proportion. Nombre de propositions de la CSSS sont en contradiction avec le principe de concurrence et, par ailleurs, avec l'article de loi voulant améliorer la situation sur le plan des données.

Pour toutes ces raisons, la Fédération des médecins suisses (FMH) s'oppose de manière catégorique à une levée de l'obligation de contracter qui laisserait le choix des médecins autorisés à contracter, comme le voulait le Conseil des Etats, aux seuls assureurs-maladie. Cela représenterait un changement de système d'une telle importance qu'il exigerait un référendum populaire. Il serait plus raisonnable que le Parlement entreprenne les démarches nécessaires à la mise en application de la LAMal actuellement valable. Il s'agit en particulier de définir avec précision le catalogue des prestations obligatoires, les critères d'efficacité, d'adéquation et d'économicité ainsi que les conditions légales permettant aux associations professionnelles d'exclure de leurs conventions des membres non qualifiés. Ces mesures permettront de satisfaire à la majorité des exigences posées par les Chambres.

Dr Hans Heinrich Brunner Président de la FMH 